\title{
Translation and Validation of Patient Satisfaction with Pharmacist Services Questionnaire (PSPSQ 2.0)
}

\author{
Mohamed Azmi Hassali ${ }^{1}$, Fahad Saleem ${ }^{2}$, Ashutosh Kumar Verma ${ }^{1, *}$, Weng Yan Choy ${ }^{1}$, Ahmed Ibrahim Nouri ${ }^{1}$, Mohd Fadli Mohd Asmani ${ }^{3}$ \\ 'School of Pharmaceutical Sciences, Universiti Sains Malaysia, Penang, MALAYSIA. \\ ${ }^{2}$ Faculty of Pharmacy and Health Sciences, University of Baluchistan, Quetta, PAKISTAN. \\ ${ }^{3}$ School of Pharmacy, Management and Science University, Selangor, MALAYSIA.
}

\begin{abstract}
Objective: To assess the psychometric properties of the PSPSO 2.0, an instrument to measure patient satisfaction with pharmacist services in a clinical setting. Methods: An observational study design was conducted in Ipoh, Perak, Malaysia from February to April 2016. Permission to reuse the questionnaires was granted before testing of the same. Forward and backtranslation was conducted prior to the study for evaluating the equivalence of meaning between the original source and target texts across culture. The questionnaires were administered randomly to participants who have their subsequent visits with a pharmacist at any public or private hospital in Ipoh. Test-retest was carried out in order to evaluate the stability of test over time using Intra-Class Coefficient. Reliability of questionnaire was tested using Cronbach's alpha coefficient. Collected data were analyzed for their consistency and validity using confirmatory factor analysis. Results: There were 130 respondents for the first time point and 80 respondents during retest. About $60 \%$ of the respondents were female; $51.5 \%$ were
\end{abstract}

aged 47 and above; mostly Chinese and almost half of the respondents worked in the government sector. The ICC for most items tested for intrarater (test-retest) reliability was good with a majority of items exhibiting coefficients $>0.80$. Conclusion: The results suggest that the PSPSO 2.0 can serve as a reliable and valid instrument to measure patient satisfaction with clinical services provided by pharmacists.

Key words: Patient satisfaction, Pharmacy services, Translation, Validation. Correspondence

Mr. Ashutosh Kumar Verma, School of Pharmaceutical Sciences, Universiti Sains Malaysia, Penang, MALAYSIA.

Phone: +60-111 8922437

Email: ashutoshverma.med@gmail.com

DOI: 10.5530/jyp.2018.10.94

\section{INTRODUCTION}

Patient satisfaction with health care services has gained increased attention in the last decades. It is a proxy yet effective indicator to measure the quality of healthcare services. ${ }^{1}$ Satisfaction can be defined as the extent of an individual's experience compared with his or her expectations. Patients' satisfaction is related to the extent to which general health care needs and condition-specific needs are met. ${ }^{2}$ It is not a unitary concept but rather a distillation of perceptions and values. Perceptions are patients' beliefs about occurrences. They reflect what happened. Values are the weights patients apply to those occurrences. They reflect the degree to which patients consider specific occurrences to be desirable, expected, or necessary. ${ }^{3}$ Thus, patient satisfaction towards health care services can be affected by many factors because each individual is subjective to their thoughts and values. Likewise, the healthcare providers are also subjective when giving their services.

As patient satisfaction will affect therapeutic outcomes and patient retention, evaluating to what extent patients are satisfied with health services is clinically relevant. Studies have shown that satisfied patients are more likely to comply with treatment, take an active role in their own care, to continue using medical care services and stay within a health provider and maintain a specific system. ${ }^{2}$ Patients with high health care satisfaction have been reported to result in improved health outcomes because they treasure their relationship with their healthcare providers, which increase their acceptance and adherence to medication and counselling. In recent years, patient satisfaction has also evolved as an indicator for pharmacist services. Pharmacists play a significant role in enhancing patient care and promote wellness especially in chronic cases. ${ }^{4}$ Pharmacist responsibilities include a range of care for patients, from dispensing medications to monitoring patients' health and progress to optimize their response to medication therapies. A good patient-pharmacist relationship brings concordance and improves patient adherence to medications and counselling. Patient satisfaction is affected by care services provided by pharmacists such as disease management, medication therapy adherence clinic (MTAC), pain management, emergency cases, oncology and total parental nutrition support services. ${ }^{1}$ Attitude and courtesy of pharmacists also influence patients' satisfaction. Patients will gain positive or negative perception from their medication experience, which develops patient satisfaction and set as an important factor in determining the value of healthcare services. ${ }^{5}$

Amidst the truths and significance of patient satisfaction, the question "Is patient satisfaction worth measuring?" emerged. On one side of an aisle are proponents of patient-cantered care, on the other sides are sceptics who believe that focusing on patient satisfaction will divert attention and should instead concern more on the technical quality such as appropriateness of criteria and outcome models. ${ }^{3}$ Nevertheless, helping patients achieve their goals is a fundamental aim of medicine. Because patients' goals and values vary widely, the only way to determine what patients want and whether their needs are being met is to ask them. The issue is not just on whether measuring patient satisfaction is important, but on whether satisfaction can be measured reproducibly and meaningfully. 


\section{Instrument Development (PSPSQ 2.0)}

A measurement tool named Patient Satisfaction Questionnaire (PSQ) was developed by Ware and colleagues in 1983 to assess patient satisfaction with health care services. It was an instrument that has undergone psychometric evaluation. ${ }^{6}$ As a result, the PSQ served as a framework for the development of future patient satisfaction instruments. ${ }^{1}$ The initial measure consisted of 80 items but was revised to 50 items tapping global satisfaction with medical care as well as the satisfaction of six aspects of care: technical quality, interpersonal manner, communication, financial aspects of care, time spent with physicians and accessibility of care. ${ }^{7}$ Several items of the PSQ were adapted by MacKeigan and Larson in the development and validation of a multidimensional measure of patient satisfaction with pharmacy services. ${ }^{1,6,8}$ Dimensions included by MacKeigan and Larson were an explanation, consideration, technical competence, financial aspects, accessibility, drug efficacy, over the counter (OTC) product availability, and quality of drug product. ${ }^{1,9}$

Over the past three decades, a literature review showed that there have been numerous instruments used to measure patient satisfaction towards pharmacist services. However, these instruments have several limitations. They were either a set of 3-4 questions with a specific clinic, lacking comprehensive psychometric testing, not reproducible, showed a variety of response scale or the instruments did not focus primarily on measuring patient satisfaction. These factors have limited the usage of instruments by researchers. ${ }^{1}$

The Patient Satisfaction with Pharmacist Clinical Services Questionnaire (PSPSQ) underwent two phases of development. The first phase was tested in a sample of 70 pharmacy students for comprehensibility and internal consistency, which led to refinement of several items in the second phase, yielding the final version PSPSQ 2.0. ${ }^{1}$ Collins stated that questionnaires are useful for measuring patients' reactions to improvements or changes to services. It is the most common type of instrument used for validation process. ${ }^{10}$

PSPSQ 2.0 has been evaluated psychometrically as an effective instrument and it has been commonly used by many researchers and healthcare providers in measuring patient satisfaction towards the services. ${ }^{1}$ The primary objective of this study is to translate and validate PSPSQ 2.0 in measuring patient satisfaction with the clinical services provided by pharmacists in Ipoh.

\section{MATERIALS AND METHODS}

\section{Study design}

An observational study design was carried out to psychometrically test the validity of PSPSQ 2.0. The purpose of this study was also to explore the underlying relationship between the measured variables which contributed to patient responses on pharmacist clinical services.

\section{Forward and Back-translation}

Permission to reuse the questionnaires was granted before testing the questionnaire from authors of Sakharkar et al. (2015). ${ }^{1}$ Prior to the study, forward and back-translation was carried out to evaluate the equivalence of meaning and quality between original source and target text in crosscultural research. ${ }^{11-12}$ The questionnaire was translated forwardly into Bahasa Malaysia, back-translated to English and assessed by three different experts from the School of Languages and Translation Universiti Sains Malaysia. The expert panels were all double-blinded and they had no knowledge about the questionnaire. The Bahasa Malaysia version of the questionnaire was used for administration to participants.

\section{Data collection}

This study was conducted in Ipoh, Perak, Malaysia. Following informed consent, the questionnaires were administered randomly to participants who have their subsequent visits with a pharmacist at any public or private hospital in Ipoh. Data collection was conducted at two-time points in February 2016 and continued until April 2016. The sample size was 130 respondents for the first time point and 80 respondents during retest.

\section{Statistical analyses}

There are many guidelines regarding the sample size for factor analysis, which vary across literature and researchers. According to the subjectto-variables ratio, there is a suggestion of 5-10 participants per variable up to a total of $300 .^{13-14}$ By using a ratio of 5 subjects to 1 variable, the calculated sample size was $80 .{ }^{15}$ Considering a $20 \%$ of non-participation rate, a minimum sample of 96 subjects was needed. A minimum of 120 participants is needed in factor analysis. ${ }^{16}$ Therefore, 130 subjects in this study were sufficient to generate good factor solutions.

Data entry and analysis was performed using SPSS version 22 . The statistical significance was set at $p<0.05$ (two-tailed). Demographic data was presented in numbers and percentages. Psychometric analyses included testing for reliability and construct validity. The internal consistency of tool was assessed using Cronbach's Alpha reliability analysis. It ranges from 0 to 1 for a completely unreliable test to for a completely reliable test. ${ }^{16}$ For reliability test in this questionnaire, Cronbach's alpha coefficient $\geq 0.70$ was applied. ${ }^{17}$

The stability of the construct measures was established by test-retest at an interval of 1 week by the same subjects using Intra-Class Coefficient (ICC). ${ }^{18}$ The sample size for retesting in this study was 80 and this was appropriate according to McMillan and Hanson (2014), the subject sample sizes for test-retest vary from about 40 to 480 subjects depending on the minimum acceptable error rates of the clinical protocol. ${ }^{19}$ The ICC model used was the One Way Random-effects model. Using the standards suggested by Portney and Watkins (2015), the following statistical significance was referred; ICC $<0.50$ (low), ICC: $0.50-0.75$ (moderate); ICC > 0.75 (good). ${ }^{18}$

\section{Confirmatory Factor Analysis (CFA)}

Confirmatory factor analysis was used to test the validity of questionnaire by examining the correlations between variables and factors. ${ }^{17}$ There are two main issues to consider in determining whether a particular data set is suitable for factor analysis: sample size and strength of the relationship between variables or items. ${ }^{17}$ While there is different agreement among authors in regard to how large a sample size should be, Hinton et al. (2014) mentioned that a minimum of 120 participants is needed in factor analysis. ${ }^{16}$ The strength of relationship among variables is determined by using Kaiser-Meyer-Olkin (KMO) and Bartlett's test of sphericity. ${ }^{17}$ If the KMO test comes out at 0.6 or higher, the data is suitable and can be used continuously for confirmatory factor analysis. ${ }^{16-17} \mathrm{KMO}$ values $>0.8$ are considered good and serve as an indication that factor analysis will be useful for the variable. On the other hand, Bartlett's test of sphericity should be significant which indicates that it is appropriate and worth continuing with factor analysis as there are relationships to investigate. ${ }^{17}$ Principal axis factoring method was used in factor extraction because the data collection was not normally distributed. ${ }^{17}$ In factor rotation and interpretation, Oblimin Oblique rotation method was used to show the correlation among factors. ${ }^{17}$ A rule of thumb is that the factor loading should be 0.3 or greater. The higher the factor loading, the more important that variable is to the factor. ${ }^{16-17}$

Sakharkar et al. suggested to exclude the overall satisfaction items that failed to be defined as a separate construct from the future analysis, 
hence, all items related to overall satisfaction domain were not included in this study, shortening the original 20 items to 16 items. ${ }^{1}$ The final questionnaire contained 16 items of which 10 items represented the Quality of Care construct and 6 items were from Interpersonal Relationship (Pharmacist-Patient) construct.

\section{RESULTS}

\section{Respondent characteristics}

Table 1 depicts the characteristics and descriptive information for the 130 respondents. $60 \%$ of the respondents were female, $51.5 \%$ were aged 47 and above. Among the respondents $70 \%$ were Chinese, $13.8 \%$ were Malay and $11.5 \%$ were Indian. Majority $(86.2 \%)$ of respondents had educational level until college or university. More than half of the respondents $(53.8 \%)$ worked in the government sector. Over threefourth $(77.7 \%)$ of the respondents were staying with family members and $40.8 \%$ of them had monthly income more than MYR5000.

\section{Psychometric properties of the instrument}

The reliability of the questionnaire was established by testing both consistency and stability. In Table 2, the Quality of Care (QOC) construct with 10 items exhibited Cronbach's alpha values, $\alpha=0.907$ (Test) and $\alpha=0.883$ (Retest) at two-time points. The Interpersonal Relationship (IPR) construct with 6 items had lower alpha values at $\alpha=0.762$ (Test) and $\alpha=0.672$ (Retest). However, the alpha values for the pooled 16 items were $\alpha=0.913$ (Test) and $\alpha=0.880$ (Retest) respectively, which has exceeded the pre-set value (0.70) and illustrated very good reliability within the constructs. The Intra-class Correlation Coefficient (ICC) calculated using One Way Random Model (Model 1) with single mea-

Table 1: Respondents' characteristics and descriptive statistics $(n=130)$.

\begin{tabular}{|c|c|c|}
\hline \multicolumn{2}{|c|}{ Respondents' characteristics ( $n=130$ ) } & \multirow{2}{*}{$\begin{array}{c}\text { Frequency (\%) } \\
78(60)\end{array}$} \\
\hline Cendr & Female & \\
\hline Gender & Male & $52(40)$ \\
\hline \multirow{4}{*}{ Age } & $18-27$ & $24(18.5)$ \\
\hline & $28-37$ & $19(14.6)$ \\
\hline & $38-47$ & $20(15.4)$ \\
\hline & $>47$ & $67(51.5)$ \\
\hline \multirow{4}{*}{ Ethnic } & Malay & $18(13.8)$ \\
\hline & Chinese & $91(70)$ \\
\hline & Indian & $15(11.5)$ \\
\hline & Others & $6(4.6)$ \\
\hline \multirow{3}{*}{ Educational Level } & Primary school & $2(1.5)$ \\
\hline & Secondary school & $16(12.3)$ \\
\hline & College / university & $112(86.2)$ \\
\hline \multirow{4}{*}{ Occupation } & Government sector & $70(53.8)$ \\
\hline & Private sector/self-employed & $30(23.1)$ \\
\hline & Retired & $8(6.2)$ \\
\hline & Student & $22(16.9)$ \\
\hline \multirow{3}{*}{ Living status } & Staying alone & $27(20.8)$ \\
\hline & With family members & $101(77.7)$ \\
\hline & With non-family members & $2(1.5)$ \\
\hline \multirow{6}{*}{$\begin{array}{c}\text { Income level } \\
\text { (MYR=Malaysia } \\
\text { Ringgit) }\end{array}$} & $<$ MYR 1000 & $10(7.7)$ \\
\hline & MYR 1001 - MYR 2000 & $3(2.3)$ \\
\hline & MYR 2001 - MYR 3000 & $16(12.3)$ \\
\hline & MYR 3001 - MYR 4000 & $27(20.8)$ \\
\hline & MYR 4001 - MYR 5000 & $21(16.2)$ \\
\hline & $>$ MYR 5001 & $53(40.8)$ \\
\hline
\end{tabular}

Table 2: Pilot Test: Cronbach's Alpha values at two-time points $(n=30)$.

\begin{tabular}{cccc} 
& & & \multicolumn{2}{c}{$\begin{array}{c}\text { Cronbach's Alpha Coefficient } \\
\text { Constructs }\end{array}$} & $\begin{array}{c}\text { Number of } \\
\text { items }\end{array}$ & \begin{tabular}{c} 
(based on standardized items) \\
\cline { 3 - 4 }
\end{tabular} & & $\begin{array}{c}\text { Test (Week 1) } \\
\mathrm{n}=30\end{array}$ & $\begin{array}{c}\text { Test (Week 2) } \\
\mathrm{n}=30\end{array}$ \\
\hline $\begin{array}{c}\text { Quality of Care (QOC) } \\
\text { Interpersonal Relationship } \\
\text { (IPR) }\end{array}$ & 10 & 0.907 & 0.883 \\
$\begin{array}{c}\text { Pooled (all 16 items) } \\
\text { (1) }\end{array}$ & 6 & 0.762 & 0.672 \\
\hline
\end{tabular}

surements is shown in Table 3. The ICC for most items tested for intra-rater (test-retest) reliability was good with a majority of items exhibiting coefficients $>0.80$. Hence, there is evidence for the repeatability for construct measurements between two-time points.

\section{Reliability and validity}

For reliability testing and factor analysis, four of the survey items were excluded due to their low-reliability values $(<0.50)$. These items were "I was satisfied with the overall care provided by my pharmacist." and "I would recommend my pharmacist to people I know." and "If needed, I would continue seeing this pharmacist for my healthcare needs." and "rating on expectations to the overall care provided by the pharmacist."

\section{Confirmatory factor analysis: Construct validity}

A factor loading is a degree to which every variable correlates with a factor (Burgess, 2006). If a factor loading is high (above 0.3 ) or very high (above 0.6 ), then the relevant variable helps to describe that factor quite well. Factor loadings below 0.3 may be ignored. In this study, the item IPR4 "The pharmacist was respectful to me during our interactions" had extracted communalities of only 0.103 and a factor loading of 0.318 in factor matrix, which was inaccurately loaded into QOC construct, suggesting that this item be removed in the future. Table 4 shows complete survey items, communalities and rotated factor loading.

In conclusion, Confirmatory Factor Analysis resulted that ten items QOC fall into the factor of Quality of Care. Certain IPR items were somewhat confusingly loaded between factor QOC and IPR. The questions were suggested could be improved, rephrased or reconstructed to allow IPR items to be distinguishable from QOC.

\section{DISCUSSION}

This study accessed the psychometric properties of PSPSQ 2.0, a tool to measure patient satisfaction with pharmacist services rendered in a clinical setting. Permission to reuse the questionnaire from Sakharkar et al. was granted before testing of questionnaires in Ipoh, Perak, Malaysia. ${ }^{1}$ The aspects of quality of care and interpersonal relationship between pharmacist and patient were measured. There were a total of twenty survey items but four items were excluded from the component analysis to avoid confusion. However, as a whole, the scale for the entire survey demonstrated validity and reliability.

Item analyses of PSPSQ 2.0 had considerable consistent data with Cronbach's alpha values above pre-test value and ICC $>0.75$. Hence, this showed reliability and stability of the instrument. Patients who took part in the study were generally moderately satisfied with the quality of care and relationship with their pharmacist. They were contented with the professionalism of clinical pharmacists in terms of their knowledge and giving information, yet they thought that the clinical pharmacists did not show enough commitment to follow up patients on a continuous basis. 
Table 3: Reliability of Test-retest ( $\mathbf{n}=\mathbf{3 0}$ ) using Intra-class Correlation Coefficient (ICC).

\begin{tabular}{|c|c|c|c|c|}
\hline Survey Items & Code & $\begin{array}{l}\text { Intra-class } \\
\text { Correlation } \\
\text { Coefficient* }\end{array}$ & $\begin{array}{c}95 \% \\
\text { Confidence } \\
\text { Interval }\end{array}$ & p-value \\
\hline QOC1 The pharmacist fully addressed the main health reason/concerns/issues during my visit. & $\operatorname{ICC}[1,1]$ & 0.840 & $0.693-0.920$ & $<0.05$ \\
\hline QOC2 The pharmacist was professional in all of our interactions. & $\operatorname{ICC}[1,1]$ & 0.899 & $0.798-0.950$ & $<0.05$ \\
\hline QOC3 The pharmacist explained information to me in a manner that I could understand. & $\operatorname{ICC}[1,1]$ & 0.829 & $0.674-0.914$ & $<0.05$ \\
\hline QOC4 The pharmacist checked to see if I understood all the information. & $\operatorname{ICC}[1,1]$ & 0.918 & $0.836-0.960$ & $<0.05$ \\
\hline QOC5 The pharmacist spent as much time necessary to help me with my questions and concerns. & $\operatorname{ICC}[1,1]$ & 0.986 & $0.732-0.931$ & $<0.05$ \\
\hline QOC6 The pharmacist made sure I understood how important it is to follow the drug regimen. & $\operatorname{ICC}[1,1]$ & 0.879 & $0.764-0.941$ & $<0.05$ \\
\hline QOC7 The pharmacist provided useful recommendations on how to take my medications. & $\operatorname{ICC}[1,1]$ & 0.858 & $0.726-0.930$ & $<0.05$ \\
\hline $\begin{array}{c}\text { QOC8 The pharmacist provided useful recommendations about managing my overall health (e.g. diet, } \\
\text { exercise). }\end{array}$ & $\operatorname{ICC}[1,1]$ & 0.860 & $0.729-0.931$ & $<0.05$ \\
\hline $\begin{array}{c}\text { QOC9 The pharmacist worked with me to manage my medication-related issues (e.g. cost, side effects of } \\
\text { drugs). }\end{array}$ & $\operatorname{ICC}[1,1]$ & 0.844 & $0.700-0.922$ & $<0.05$ \\
\hline QOC10 The pharmacist followed up on my progress in a timely manner. & $\operatorname{ICC}[1,1]$ & 0.900 & $0.802-0.951$ & $<0.05$ \\
\hline IPR1 The pharmacist was caring and kind in dealing with my health issues. & $\operatorname{ICC}[1,1]$ & 0.823 & $0.664-0.911$ & $<0.05$ \\
\hline IPR2 The pharmacist encouraged me to achieve my treatment goals. & $\operatorname{ICC}[1,1]$ & 0.938 & $0.874-0.970$ & $<0.05$ \\
\hline IPR3 I felt comfortable in my interactions with the pharmacist. & $\operatorname{ICC}[1,1]$ & 0.712 & $0.481-0.851$ & $<0.05$ \\
\hline IPR4 The pharmacist was respectful to me during our interactions. & $\operatorname{ICC}[1,1]$ & 0.803 & $0.630-0.901$ & $<0.05$ \\
\hline IPR5 The pharmacist was committed to improving my health. & $\operatorname{ICC}[1,1]$ & 0.869 & $0.745-0.935$ & $<0.05$ \\
\hline IPR6 I could trust the information that the pharmacist provided. & $\operatorname{ICC}[1,1]$ & 0.851 & $0.712-0.926$ & $<0.05$ \\
\hline
\end{tabular}

Note: ICC ${ }^{\star}$ values using One Way Random effect model (Model 1), single measures, 95\% Confidence Interval

Table 4: Survey Items, Communalities and Rotated Factor Loading ( $n=130)$.

\begin{tabular}{|c|c|c|c|c|c|c|c|}
\hline \multirow{4}{*}{ Survey Items } & \multirow{3}{*}{\multicolumn{2}{|c|}{$\begin{array}{c}\begin{array}{c}\text { Factor } \\
\text { Matrix }^{\mathrm{a}}\end{array} \\
\text { Factor } \\
\text { Loading }\end{array}$}} & \multirow{3}{*}{\multicolumn{2}{|c|}{$\begin{array}{c}\begin{array}{c}\text { Structure } \\
\text { Matrix }\end{array} \\
\text { Factor } \\
\text { Loading }\end{array}$}} & \multirow{3}{*}{\multicolumn{2}{|c|}{$\begin{array}{c}\text { Pattern } \\
\text { Matrix }^{\mathrm{b}}\end{array}$}} & \multirow{4}{*}{$\begin{array}{c}\text { Communalities } \\
\text { Extraction }\end{array}$} \\
\hline & & & & & & & \\
\hline & & & & & & & \\
\hline & QOC & IPR & QOC & IPR & QOC & IPR & \\
\hline $\begin{array}{l}\text { QOC1 The pharmacist fully addressed the main health reason /concerns /issues during my } \\
\text { visit. }\end{array}$ & .631 & .111 & .527 & .537 & .378 & .394 & .411 \\
\hline QOC2 The pharmacist was professional in all of our interactions. & .644 & -.071 & .615 & .421 & .532 & .220 & .420 \\
\hline QOC3 The pharmacist explained information to me in a manner that I could understand. & .460 & -.418 & .592 & .050 & .669 & -.203 & .386 \\
\hline QOC4 The pharmacist checked to see if I understood all the information. & .650 & -.323 & .725 & .253 & .734 & -.024 & .527 \\
\hline $\begin{array}{l}\text { QOC5 The pharmacist spent as much time necessary to help me with my questions and } \\
\text { concerns. }\end{array}$ & .685 & -.492 & .828 & .163 & .894 & -.174 & .711 \\
\hline QOC6 The pharmacist made sure I understood how important it is to follow the drug regimen. & .737 & -.413 & .842 & .255 & .869 & -.073 & .713 \\
\hline QOC7 The pharmacist provided useful recommendations on how to take my medications. & .674 & -.113 & .660 & .415 & .587 & .193 & .467 \\
\hline $\begin{array}{l}\text { QOC8 The pharmacist provided useful recommendations about managing my overall health } \\
\text { (e.g. diet, exercise). }\end{array}$ & .677 & .246 & .512 & .662 & .306 & .547 & .519 \\
\hline $\begin{array}{l}\text { QOC9 The pharmacist worked with me to manage my medication related issues (e.g. cost, side } \\
\text { effects of drugs). }\end{array}$ & .724 & .101 & .615 & .598 & .455 & .426 & .535 \\
\hline QOC10 The pharmacist followed up on my progress in a timely manner. & .721 & .083 & .620 & .583 & .467 & .407 & .526 \\
\hline IPR1 The pharmacist was caring and kind in dealing with my health issues. & .559 & .713 & .210 & .895 & -.149 & .952 & .820 \\
\hline IPR2 The pharmacist encouraged me to achieve my treatment goals. & .660 & .138 & .542 & .577 & .379 & .434 & .455 \\
\hline IPR3 I felt comfortable in my interactions with the pharmacist. & .427 & .505 & .177 & .656 & -.083 & .688 & .437 \\
\hline IPR4 The pharmacist was respectful to me during our interactions. & .318 & .046 & .270 & .264 & .199 & .189 & .103 \\
\hline IPR5 The pharmacist was committed to improving my health. & .566 & -.176 & .588 & .293 & .557 & .083 & .351 \\
\hline IPR6 I could trust the information that the pharmacist provided. & .405 & .432 & .187 & .591 & -.041 & .607 & .351 \\
\hline
\end{tabular}

Note: QOC=Quality of Care; IPR=Interpersonal Relationship; KMO $=0.834$, Barlett's Test of Sphericity, $\mathrm{p}<0.05$

Extraction Method: Principal Axis Factoring; Rotation Method: Oblimin with Kaiser Normalization

a) 2 factors extracted. 9 iterations required. b) Rotation converged in 9 iterations. 
This implied the interpersonal relationship between pharmacist and patient is yet to be improved.

The results of confirmatory factor analysis supported the validity of PSPSQ 2.0 questionnaire. KMO measure of sampling adequacy for the factor analysis was 0.834 which signified the success of factorial analyses. Bartlett test was significant which indicated that it was worth continuing with the factor analysis as there were relationships to investigate. Our survey with 130 total respondents has contributed to the suitability of using factor analysis which was supported by the recommendation of minimum 120 participants by Hinton et al. ${ }^{16}$ This survey demonstrated reliability for being consistent and stable in Cronbach's alpha values. It ranges from 0 for a completely unreliable test to 1 for a completely reliable test. All values of the survey items exhibited reliable values which were nearing to 1: Quality of Care (QOC) construct with 10 items exhibited Cronbach's alpha values, $\alpha=0.907$ (Test) and $\alpha=0.883$ (Retest) at two time points; the Interpersonal Relationship (IPR) construct with 6 items had alpha values at $\alpha=0.762$ (Test) and $\alpha=0.672$ (Retest); the pooled 16 items have values $\alpha=0.913$ (Test) and $\alpha=0.880$ (Retest). The ICC for most items tested for intra-rater (test-retest) reliability was good with a majority of items exhibiting coefficients $>0.80$. Hence, it was evident for the repeatability for construct measurements between twotime points.

The perception of a service is one of the most important drivers of patient satisfaction. Their perception is affected by the interaction, communication, professionalism and attitude of pharmacists. Daily interaction between pharmacists and patients may improve current trends in service delivery or open avenues for expectations between two parties. ${ }^{20}$ Patients frequently seek information from pharmacists on medication use, dosage, drug administration, side effects and drug interaction. In this study, most of the patients agreed that pharmacist gave an impression as polite and respectful personnel, caring and kind in dealing with their health issues. They felt comfortable when interacting with pharmacists. Patients were generally putting their trust on pharmacists and believed in the information given by them because the public see them as the professionals. On the other side, patients did not agree with the survey items "the pharmacist encouraged me to achieve my treatment goals" and "the pharmacist was committed to improving my health", reflecting a lacking of good patient-pharmacist interpersonal relationship in the pharmaceutical healthcare system. This factor is a hindrance to many therapeutic failure or complications and non-compliance among patients. A good patient-pharmacist-relationship can improve the medication adherence among patients significantly, besides improving their awareness and understanding towards diseases, thus exerting a better control over chronic diseases such as diabetes and coronary artery disease.

Demographic factors that may affect patient satisfaction with pharmacist services include educational level, occupation and income level, and personal behaviour. Quality of patient care depends directly on the quality of patient education and responsibility. Patients with higher educational level, who have acknowledgment about their rights, will definitely influence their expectations of quality services. For instance, if people know about their rights of getting medication choice and uses, they would expect more from their caregivers and consequently the quality of healthcare services increases. More educated patients have more realistic expectations of the healthcare providers. A pharmacist may unconsciously explain more to an educated patient and assume that the patient would understand better. ${ }^{21}$ Patients with higher educational level are also more open-minded and can accept non-conventional healthcare services. Some patients are so much bounded to their past living cultures which would have shaped their perceptions, leading them to reject some interventions provided by pharmacists or health providers. For instance, some patients have been living or growing up in a "medicines cure all" culture. They will ask their doctors to prescribe medicines regardless of any condition. They believe that they will not be healed unless they take medicines. Thus when a pharmacist insists on not giving medication without knowing the patient's background, it will provoke patient dissatisfaction to the service. ${ }^{21}$

Patients' financial status may also affect the quality of healthcare services. Income status of an individual is correlated with their occupational status. According to the Statistics Department of Malaysia (2014), the increase of household expenditure was more significant than the increase in average income. Malaysian's health expenditure has two-fold increase in gross domestic product (GDP) from 2.94\% (1997) to 4.53\% (2013). Statistics showed a total of RM23.35 billion and RM21.50 billion were spent respectively in government and private healthcare setting in $2013 .{ }^{22}$ However, with the implementation of GST and impact of currency exchange rates, this may affect the consumers' purchasing power and limit their choice of healthcare services. Patients who cannot afford a better quality treatment cost may end up being arbitrary to alternative treatment, thus affecting their satisfaction towards pharmacist service due to their negative perception of the whole healthcare system.

The patient's attitude and behaviour also affect the attitudes of caregivers. If a patient behaves himself or herself, care-givers unintentionally provide better services. Studies showed that a polite and eloquent patient will receive better services from healthcare providers. Patient recognition may also influence the quality of provided services. A patient that acknowledges a pharmacists' or care-givers' effort will motivate the personnel to do better in their service. This shows that patient cooperation and involvement is needed in fostering a mutual relationship with the care providers. ${ }^{21}$

This survey may help health professionals and pharmacists to identify potential areas for service improvement and health expenditure may be optimised through patient-guided planning and evaluation. PSPSQ 2.0 is beneficial to assess patient satisfaction with pharmacist services because this is a cost-effective method, practical and scalable. This instrument is reproducible and psychometric tested, which can be up taken by researchers.

\section{CONCLUSION}

The PSPSQ 2.0 is an instrument developed to assess patient satisfaction with various pharmacist clinical services. Results from this survey suggested that the PSPSQ 2.0 can be used as a valid and reliable tool to measure patient satisfaction with pharmacist clinical services.

\section{LIMITATION}

This study has some limitations that should be taken into account when extrapolating the results. The sampling method may have introduced selection bias because three-quarter of the respondents were from Chinese ethnicity. This is because there was $44.10 \%$ of Chinese in Ipoh according to the demographic data, which marked the highest population among the other ethnics. ${ }^{23}$ This study was also limited by the typical perception of patients towards pharmacist service, in which they carried forward their medication and counselling experiences from the past to present.

\section{ACKNOWLEDGEMENT}

I would like to acknowledge Pn. Nor Aini from School of Translation USM for her help in doing forward and back-translation of the questionnaire. I am also gratefully indebted to Mr. Vincent Gan and Ms Christine Tan Liang Hoay for their very valuable contribution in computing the 
results. Lastly, I wish to give a token of appreciation to all the anonymous respondents who have participated in this survey.

\section{CONFLICT OF INTEREST}

The authors declare no conflcit of interest.

\section{ABBREVIATIONS}

PSPSQ: Patient Satisfaction with Pharmacist Services Questionnaire; ICC: IntraClass Coefficient; MTAC: Medication Therapy Adherence Clinic; OTC: Over The Counter; CFA: Confirmatory Factor Analysis; KMO: Kaiser-Meyer-Olkin; QOC: Quality of Care; IPR: Interpersonal Relationship; GDP: Gross Domestic Product.

\section{REFERENCES}

1. Sakharkar P, Bounthavong M, Hirsch JD, Morello CM, Chen TC, Law AV. Development and validation of PSPSQ 2.0 measuring patient satisfaction with pharmacist services. Res Social Adm Pharm. 2015;11(4):487-98.

2. Asadi-Lari M, Tamburini M, Gray D. Patients' needs, satisfaction, and health related quality of life: Towards a comprehensive model. Health Qual Life Outcomes. 2004;2(1):32.

3. Kravitz R. Patient Satisfaction with Health Care: Critical Outcome or Trivial Pursuit?. J Gen Intern Med. 1998;13(4):280-2.

4. Wagner $\mathrm{EH}$. The role of patient care teams in chronic disease management Br Med J. 2000;320(7234):569.

5. Kane RL, Maciejewski M, Finch M. The relationship of patient satisfaction with care and clinical outcomes. Med care. 1997;35(7):714-30.

6. Ware JE, Snyder MK, Wright WR, Davies AR. Defining and measuring patient satisfaction with medical care. Eval Program Plann. 1983;6(3-4):247-63.

7. Marshall GN, Hays RD. The patient satisfaction questionnaire short-form (PSQ-18). Rand Santa Monica, CA. 1994.

8. Ware Jr JE, Hays RD. Methods for measuring patient satisfaction with specific medical encounters. Med Care. 1988;26(4):393-402.

9. MacKeigan LD, Larson LN. Development and validation of an instrument to measure patient satisfaction with pharmacy services. Med Care. 1989:522-36.
10. De Jesus EM, Cardoso A, Valença E, Da Fonseca FL, Santana RS, Rabelo JS, et al. Validation of tools for the evaluation of pharmaceutical services: A systematic review. Afr J Pharm Pharmacol. 2016;10(45):954-66.

11. Brislin RW. Back-translation for cross-cultural research. J Cross Cult Psychol. 1970;1(3):185-216.

12. WHO. Process of translation and adaptation of instruments: World Health Organization. 2017 [Available from: http://www.who.int/substance_abuse/research_ tools/translation/en/].

13. Tinsley HE, Kass RA. The latent structure of the need satisfying properties of leisure activities. J Leis Res. 1979;11(4):278.

14. McGuire B, Tinsley HE. A contribution to the construct validity of the Tennessee Self-Concept Scale: A confirmatory factor analysis. Appl Psychol Meas. 1981; 5(4):449-57.

15. Barrett PT, Kline P. The observation to variable ratio in factor analysis. Personality study and group behavior. 1981;1(1):23-33.

16. Hinton PR, McMurray I, Brownlow C. SPSS explained. $2^{\text {nd }}$ ed. London and Newyork: Routledge Taylor and Francis Group. 2014:387.

17. Hinton PR, McMurray I, Brownlow C. SPSS explained. (UK). 2004.

18. Portney LG, Watkins MP. Foundations of clinical research: Applications to practice: FA Davis. 2015

19. McMillan GP, Hanson TE. Sample size requirements for establishing clinical test-retest standards. Ear and Hearing. 2014;35(2):283-6.

20. Ankrah DN, Ofei-Palme CN, Nordor M, Nelson F, Ocansey D, Bruce E. Patients perception and expectations of services provided by pharmacists in Ghanaian hospitals. Afr J Pharm Pharmacol. 2014;8(46):1164-72

21. Mosadeghrad AM. Factors influencing healthcare service quality. Int J Health Policy Manag. 2014;3(2):77-89.

22. Chen Chaw Min P. Health Spending: The Malaysian Experience. Department of Statistics, Malaysia. 2014;11(2):101.

23. Department of Statistics Malaysia. Current population estimates, Malaysia, 2014 - 2016 Malaysia: Department of Statistics. 2016. [Available from: https:// www.statistics.gov.my/index.php?r=column/cthemeByCat\&cat=155\&bul_id= OWIxdEVoYIJCSOhUZzJyRUcvZEYxZz09\&menu_id=LOpheU43NWJwRWVSZk IWdzQ4TIhUUT09]

Article History: Submission Date : 04-02-2018; Revised Date : 07-04-2018; Acceptance Date : 15-04-2018.

Cite this article: Hassali MA, Saleem F, Verma AK, Choy WY, Nouri Al, Asmani MFM. Translation and Validation of Patient Satisfaction with Pharmacist Services Questionnaire (PSPSQ 2.0). J Young Pharm. 2018;10(4):427-32. 